\title{
Analysis of a 3 '-translation enhancer in a tombusvirus: A dynamic model for RNA-RNA interactions of mRNA termini
}

\author{
MARC R. FABIAN and K. ANDREW WHITE \\ Department of Biology, York University, Toronto, Ontario M3J 1P3, Canada
}

\begin{abstract}
Tomato bushy stunt virus is a (+)-strand RNA virus that is neither $5^{\prime}$-capped nor $3^{\prime}$-polyadenylated. Translation of viral proteins is instead mediated by an RNA element, the $3^{\prime}$-cap-independent translational enhancer (3'CITE), which is located in its $3^{\prime}$ untranslated region (UTR). The $3^{\prime} \mathrm{CITE}$ is proposed to recruit the translational machinery to the viral message, while a longdistance RNA-RNA interaction between the $3^{\prime}$ CITE and 5' UTR is thought to deliver the 435 ribosomal subunit to the 5' end of the viral mRNA. Here we provide the first evidence that the 5' UTR and 3'CITE interact physically. Mutational analysis showed that formation of this RNA-RNA interaction in vitro correlates well with efficient translation in vivo, thus supporting its functional relevance. Other analyses of the $3^{\prime}$ CITE confirmed an overall Y-shaped RNA secondary structure and demonstrated the importance of numerous minor structural features for efficient translation of viral mRNAs. Functional studies on the role of the 5' UTR revealed that despite the absence of a cap structure, 435 subunits load at the very $5^{\prime}$ end and scan in a $3^{\prime}$ direction. These results indicate that the $5^{\prime}-3^{\prime}$ RNA-RNA interaction is likely disrupted by scanning ribosomal subunits and suggest a dynamic model for the interaction of mRNA termini during active translation.
\end{abstract}

Keywords: RNA structure; RNA virus; plant virus; tombusviridae; TBSV

\section{INTRODUCTION}

After a (+)-strand RNA virus has successfully entered a host cell, it must quickly begin translating its encoded proteins in order to initiate an infection. In eukaryotic systems, many $(+)$-strand RNA viruses contain the same $5^{\prime}$-cap and a $3^{\prime}$-poly(A) tail structures that are present at the termini of most cellular mRNAs (Dreher and Miller 2006). These structures function synergistically to mediate translation by interacting via a protein bridge consisting minimally of translation initiation factors eIF4E and eIF4G, and poly(A) binding protein (PABP). The eIF4E-eIF4G-PABP complex is linked to the $5^{\prime}$-cap by eIF4E, while the PABP mediates the interaction with the $3^{\prime}$-poly(A) tail (Wells et al. 1998). This protein-mediated circularization of mRNAs acts to facilitate efficient translation in eukaryotic systems (Gebauer and Hentze 2004). The robustness of translation is strongly influenced by the efficiency of the initiation step in the process. Initiation of $5^{\prime}$-cap- and $3^{\prime}$-poly(A)-

Reprint requests to: $\mathrm{K}$. Andrew White, Department of Biology, York University, 4700 Keele St., Toronto, Ontario, Canada M3J 1P3; e-mail: kawhite@yorku.ca; fax: (416) 736-5698.

Article published online ahead of print. Article and publication date are at http://www.rnajournal.org/cgi/doi/10.1261/rna.69506. dependent translation involves the small subunit of the ribosome entering at the $5^{\prime}$ end of the $5^{\prime}$ untranslated region (UTR) and then scanning in a $3^{\prime}$ direction until it encounters a start codon in a favorable context (Kozak 2002). In most cases, start codons flanked by a guanylate at position +4 and a purine at -3 (relative to the initiation codon) are well recognized by scanning subunits and, in turn, mediate efficient initiation (Kozak 2002).

(+)-Strand RNA viruses that do not contain a $5^{\prime}$-cap can compensate for this deficiency by maintaining an internal ribosome entry site in their $5^{\prime}$ UTRs that recruits ribosomes directly to the initiation codon (Pelletier and Sonenberg 1988). Other (+)-strand RNA viruses that lack both a $5^{\prime}$-cap and a $3^{\prime}$-poly(A) tail utilize a different translational strategy involving a $3^{\prime}$-proximal RNA element (Kneller et al. 2005). Members of the Tombusviridae and Luteoviridae families fall into this category and maintain RNA elements in the $3^{\prime}$ region of their genomes that act as translational enhancers, termed 3'TEs (Danthinne et al. 1993; Timmer et al. 1993; Wang and Miller 1995; Wang et al. 1997; Oster et al. 1998; Wu and White 1999; Qu and Morris 2000; Koh et al. 2002; Mizumoto et al. 2003; Shen and Miller 2004). The 3'TE of Satellite tobacco necrosis virus (STNV) has been shown to interact in vitro with canonical eukaryotic translation 
initiation factors eIF4F and eIFiso4F and thus may be involved in recruiting these complexes to the viral mRNAs in vivo (Gazo et al. 2004). Recruitment roles have also been proposed for the 3'TEs in the luteovirus Barley yellow dwarf virus (BYDV) and the tombusvirus Tomato bushy stunt virus (TBSV) (Wang et al. 1997; Wu and White 1999; Guo et al. 2000, 2001; Fabian and White 2004).

The recruitment of translational machinery to the $3^{\prime}$ end of these viral mRNAs seems counterintuitive, since translation initiates at the $5^{\prime}$ end. However, this paradox would be resolved if 3'TE-bound factors were then delivered to the $5^{\prime}$ region (Kneller et al. 2005). This event would require some form of communication between the $5^{\prime}$ and $3^{\prime}$ termini of viral mRNAs. Indeed, BYDV has been reported to form a long-distance kissing-loop interaction between RNA hairpins in its 3'TE and 5' UTR that facilitates translation (Guo et al. 2001). A similar functional 5'-3' RNARNA interaction has also been reported for TBSV (Fabian and White 2004). Furthermore, comparative sequence analysis of the genomes of members in Tombusviridae suggests that this type of $5^{\prime}-3^{\prime}$ long-distance interaction is prevalent in this large family-all of which lack $5^{\prime}$-cap and $3^{\prime}$-poly(A) tails (Fabian and White 2004). This latter observation, considered along with the fact that many members of Tombusviridae have already been shown to contain $3^{\prime}$ TEs (Kneller et al. 2005), suggests that the hypothesized role for the 5'-3' RNA-RNA interaction in delivering translational machinery to the $5^{\prime}$ end of viral mRNAs may apply to a large group of viruses.

TBSV is the prototypical member of the genus Tombusvirus in the family Tombusviridae (White and Nagy 2004). Its RNA genome is $4.8 \mathrm{~kb}$ in length, lacks both a $5^{\prime}$-cap and a $3^{\prime}$-poly(A) tail, and codes for five functional proteins (Fig. 1A; Hearne et al. 1990). The 5' -proximal p33 and its readthrough product p92 are both essential for viral RNA replication and are translated directly from the TBSV genome (Scholthof et al. 1995; Oster et al. 1998). In contrast, the $3^{\prime}$ proximally encoded proteins (coat protein [p41], movement protein [p22], and a gene silencing suppressor protein [p19]) are translated from two subge- nomic (sg) mRNAs that are produced during infections (Choi and White 2002; Lin and White 2004).

Previously, we have shown that efficient cap- and poly (A)-tail-independent translation of TBSV mRNAs in vivo requires a $3^{\prime}$-terminal RNA sequence called the $3^{\prime}$ cap-independent translational enhancer (3'CITE)
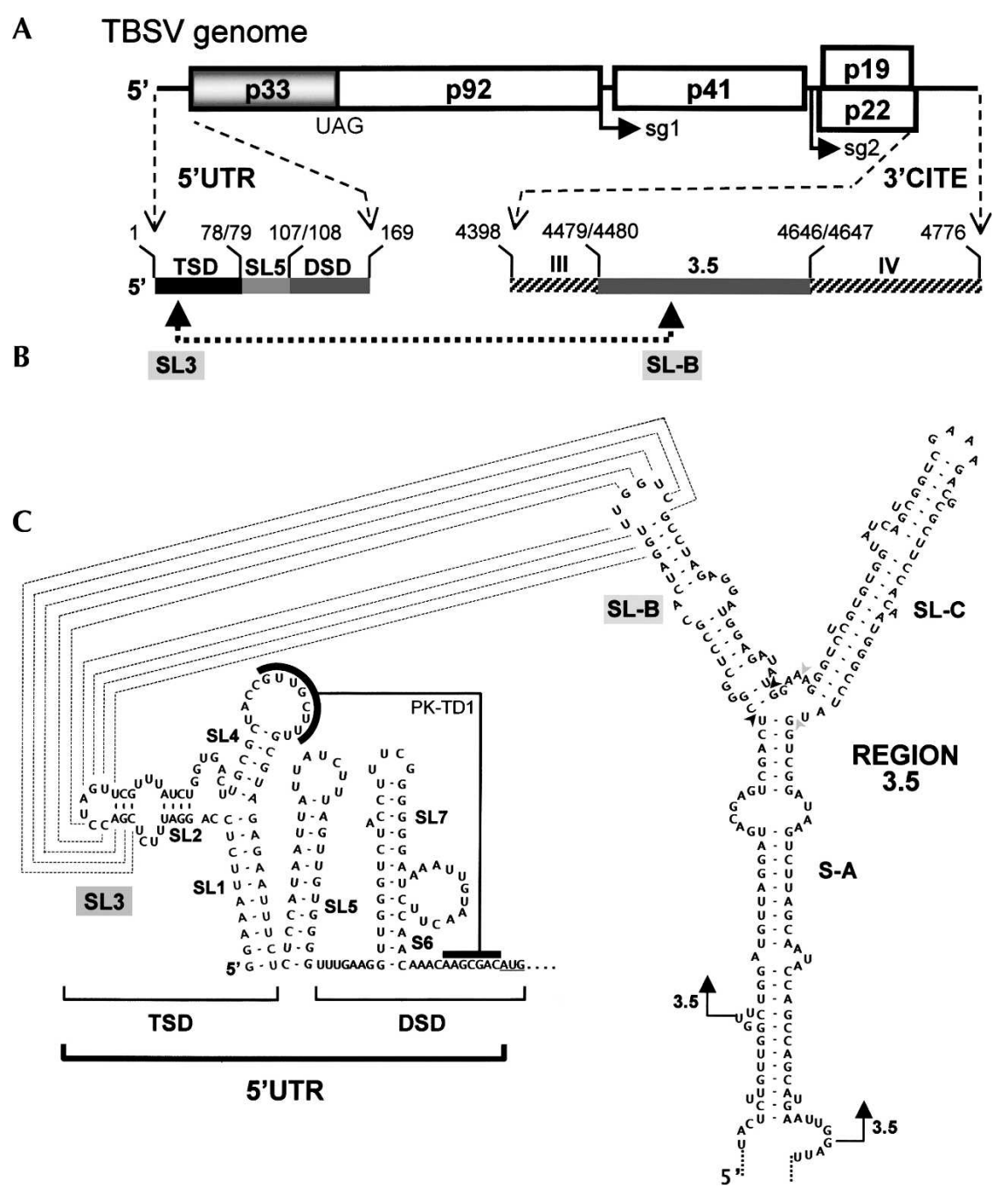

FIGURE 1. The TBSV RNA genome and its associated RNA elements. (A) A linear schematic of the TBSV RNA genome is shown with coding regions depicted as boxes (Hearne et al. 1990). The molecular masses (in thousands) of encoded proteins are prefixed with "p." Solid arrows at intergenic regions in the genome indicate the transcription initiation sites for viral $\mathrm{sg}$ mRNAs 1 and 2 ( $\mathrm{sg} 1$ and $\mathrm{sg} 2$, respectively). (B) Linear representations of $5^{\prime}$ UTR and $3^{\prime}$ CITE in the TBSV genome. Domains (TSD, SL5, DSD) and regions (III, 3.5, IV) in the $5^{\prime}$ UTR and $3^{\prime}$ CITE, respectively, are delineated in the expanded segments. Coordinates of the junctions of the various RNA segments are given on top and correspond to the numbering scheme for the TBSV genome (Hearne et al. 1990). The proposed long-distance RNA-RNA interaction between SL3 in the TSD and SL-B in R3.5 is indicated by two arrowheads connected by a dotted line. $(C)$ RNA secondary structure models for the 5' UTR and R3.5. The proposed 5'3' RNA-RNA interaction between SL3 and SL-B is shown with complementary base pairs connected by dotted lines. In the $5^{\prime}$ UTR, the interdomain base-pairing interaction between the TSD and DSD, termed PK-TD1, is indicated by thick black bars connected by a solid line. In the structure of the $3^{\prime}$ CITE, the borders of SL-B and SL-C deletion mutants (analyzed in Fig. 2B) are indicated by sets of black or gray arrowheads, respectively. The $5^{\prime}$ and $3^{\prime}$ borders of R3.5 are delineated by arrows denoted 3.5. 
(Fig. 1B; Wu and White 1999). In addition, the 5' UTR of the TBSV genome was also found to contain an RNA element, stem-loop (SL) 3, which was essential for efficient viral mRNA translation in vivo (Fig. 1B; Fabian and White 2004). The 5' UTR folds into a complex RNA structure that contains three major subelements: a T-shaped domain (TSD), a small hairpin termed SL5, and a downstream domain (DSD) (Fig. 1B,C). All three of these structures are critical for efficient viral RNA replication (Wu et al. 2001; Ray et al. 2003, 2004), whereas SL3 in the TSD represents the key element necessary for efficient TBSV mRNA translation (Fig. 1C; Fabian and White 2004).

The 3'CITE comprises three contiguous regions of the TBSV genome: region (R) III, R3.5, and RIV. Interestingly, RIII and RIV are both essential for TBSV translation and replication, whereas $\mathrm{R} 3.5$ is dispensable for viral replication but is crucial for translation (White and Nagy 2004). Mfold analysis and solution structure probing indicated that R3.5 forms a Y-shaped RNA secondary structure comprising SL-B, SL-C, and S-A (Fig. 1C; Fabian and White 2004). Deletion of the sequences corresponding to either SL-B or SL-C reduced in vivo translation of viral mRNAs by $\sim 10$-fold (Fabian and White 2004). In addition, the terminal loop of SL-B was found to be complementary to the terminal loop of SL3 in the 5' UTR and thus could potentially mediate an interaction between the $3^{\prime}$ CITE and 5' UTR (Fig. 1C). Compensatory-type mutational analysis carried out on these RNA elements in a TBSV reporter mRNA revealed a positive correlation between SL3 and SL$\mathrm{B}$ base-pairing potential and efficient translation (Fabian and White 2004). Accordingly, it was proposed that the kissing-loop interaction between SL3 and SL-B acts to mediate communication between the $3^{\prime} \mathrm{CITE}$ and $5^{\prime}$ UTR.

In the present study, we provide the first physical evidence for an interaction between the 5' UTR and 3'CITE and show that formation of the RNA-RNA complex in vitro depends on complementarity between SL3 and SL-B. In addition, the proposed Y-shaped RNA secondary structure model for R3.5 in the $3^{\prime}$ CITE was investigated by mutational analysis and shown to be functionally relevant. Moreover, we provide evidence that this $5^{\prime}$-cap-independent translational process involves ribosome loading and processive scanning from the $5^{\prime}$ end of the $5^{\prime}$ UTR. These data provide important new insights into the overall mechanism of translation in TBSV.

\section{RESULTS}

\section{The $5^{\prime}$ UTR interacts with the $3^{\prime} \mathrm{CITE}$ in vitro}

The proposed interaction between the 5' UTR and the $3^{\prime}$ CITE is supported by previous comparative sequence analysis of tombusvirus genomes, which revealed monoand covariations between complementary terminal loops of the $5^{\prime}$-proximal SL3 and $3^{\prime}$-proximal SL-B (Fabian and
White 2004). In addition, compensatory-type mutagenic studies showed that efficient translation in vivo requires maintenance of base-pairing potential between the loop sequences of these RNA adaptors (Fabian and White 2004). However, these sequence and genetic analyses were indirect and neither definitively established whether the RNA elements actually interact with each other. To address this issue, a direct approach was taken by using RNA-RNA electrophoretic mobility shift assays (EMSAs). In these studies, in vitro transcripts of the shorter $5^{\prime}$ UTR (169 nucleotides [nt]) was $5^{\prime}$-end-labeled with ${ }^{32} \mathrm{P}$ and mixed with unlabeled transcripts of the longer $3^{\prime}$ CITE $(\sim 400 \mathrm{nt})$. RNA-RNA complexes were subsequently resolved and detected by nondenaturing electrophoresis and radioanalytical scanning of gels. In these assays, the two RNAs were separately denatured and refolded before mixing, so as to promote interactions based on their native higher-order structures. Using this approach, a shifted RNA-RNA complex was readily detectable when the labeled wild-type 5' UTR was incubated with increasing concentrations of wild-type 3'CITE (Fig. 2A). An apparent dissociation constant $\left(\mathrm{K}_{\mathrm{d}}\right)$ of $\sim 600 \mathrm{nM}$ was calculated from the binding curve generated. The importance of subelements SL-B, SL-C, and R3.5 in the 3'CITE was also assessed. Deletion of either SL-B or R3.5 abolished formation of the complex, whereas the removal of SL-C had little effect (Fig. 2B). These results demonstrate that SL-B, the proposed $3^{\prime}$-adaptor in the $5^{\prime}-3^{\prime}$ interaction, is essential for efficient complex formation.

Next, the role of the complementary adapter sequences in SL3 and SL-B was investigated with respect to complex formation. This analysis was carried out by using a set of compensatory mutations shown previously to reduce and then restore translational activity in vivo (Fabian and White 2004). Two different pairs of complementary adaptor SLs, wild-type TBSV and mutant PM (Fig. 2C), were tested in homologous or heterologous combinations. 5' UTRs and 3'CITEs that contained complementary homologous SLs were able to efficiently form the complex, while noncomplementary heterologous pairs displayed obvious reductions in complex formation (Fig. 2D). Importantly, all of the EMSA results correlated well with corresponding in vivo translation activities observed previously using reporter mRNAs containing the same modifications (Fig. 2B,D, bottom row; Fabian and White 2004). These results provide compelling physical evidence for a functionally relevant interaction that is dependent on the maintenance of complementarity between the $5{ }^{\prime}$ - and $3{ }^{\prime}$-adapter SLs.

\section{Sequence and secondary structure are important for 3'CITE activity}

Next, we sought to determine if the proposed Y-shaped secondary structure of R3.5 in the $3^{\prime}$ CITE was functionally relevant. To do so, a previously established translational assay system was used that monitors translational activity 

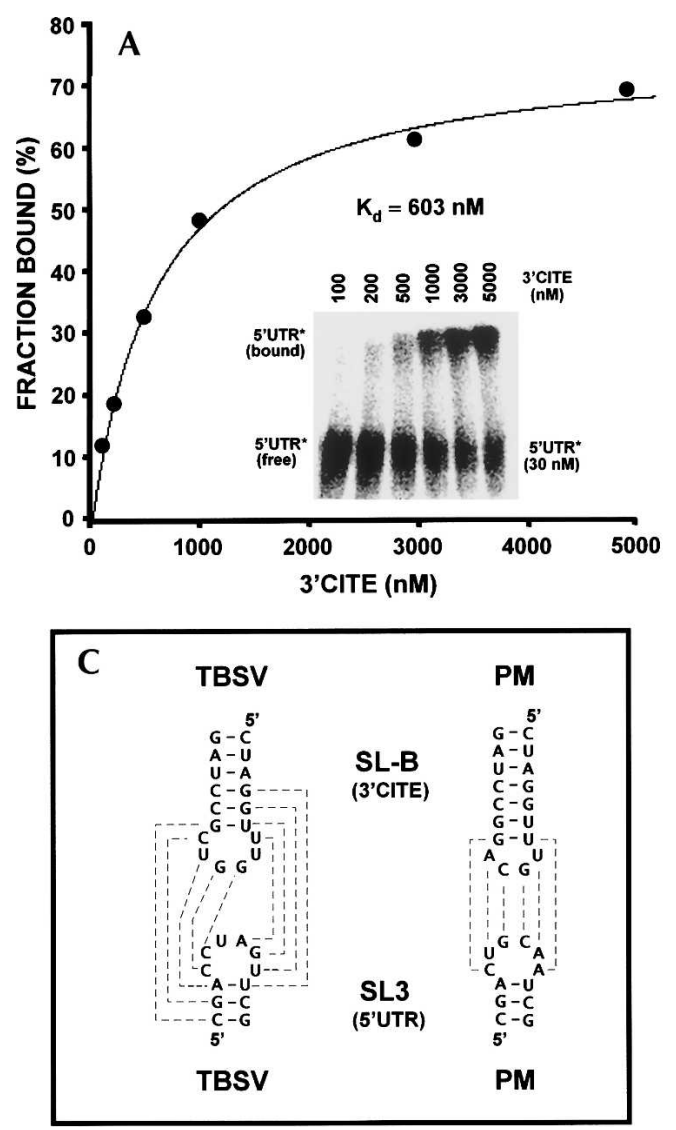

B

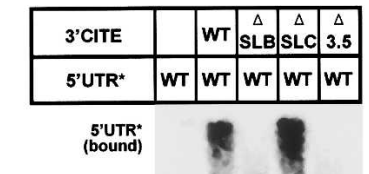

D

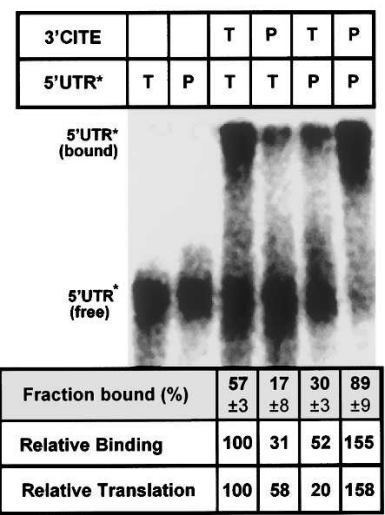

FIGURE 2. RNA-RNA binding analysis of the TBSV $5^{\prime}$ UTR and $3^{\prime}$ CITE. (A) Binding curve of $5^{\prime}$ UTR and $3^{\prime}$ CITE interaction. Labeled wild-type $5^{\prime}$ UTR $^{*}$ was incubated with increasing amounts of unlabeled wild-type $3^{\prime} \mathrm{CITE}$, and the fraction bound was plotted versus concentration. The concentrations of each used are indicated (in $\mathrm{nM}$ ). Complexes were separated in a nondenaturing gel (see inset), and the fraction of $5^{\prime} \mathrm{UTR}^{*}$ bound was quantified. Fractional values obtained were then used to generate a binding curve. Best-fit analysis was carried out using nonlinear regression analysis, and the curve generated was used to deduce an apparent $\mathrm{K}_{\mathrm{d}} \cdot(B)$ Binding analysis of $3^{\prime} \mathrm{CITE}$ mutants. The RNAs present in the binding assays are indicated at the top. Binding assays were carried out with $30 \mathrm{nM}$ wild-type $5^{\prime}$ UTR* and $1000 \mathrm{nM}$ of the indicated $3^{\prime}$ CITE. The "fraction bound" of the $5^{\prime} \mathrm{UTR}^{*}( \pm \mathrm{SD}$, from three independent experiments) is indicated at the bottom (shaded gray). The fractional values are also presented as "relative binding" levels (with the wild-type interaction set at $100 \%$ ). For comparison, the corresponding "relative translation" levels from reporter mRNAs containing the same modifications (taken from a previous study) are provided (Fabian and White 2004). The borders of deletions of SL-B and SL-C in the $3^{\prime}$ CITE are depicted in Figure 1C. $(C)$ Wildtype TBSV and mutant PM adapter SLs present in the 5' UTRs and 3'CITEs used in the binding assays in $D$. The predicted kissing-loop interaction for each homologous pair is indicated by dotted lines. (D) Binding analysis of $5^{\prime}$ UTR and $3^{\prime}$ CITE mutants containing homologous and heterologous SL pairs, as shown in C. RNAs in the binding assay contained either wild-type TBSV $(T)$ or mutant PM $(P)$ adapter SLs, as indicated at the top. Values below are as described in $B$.

indirectly via the amplification of a cotransfected viral RNA replicon (Wu and White 1999; Fabian and White 2004). In this system, the nonreplicating experimental mRNA encodes a TBSV protein, p33, which is essential for viral RNA synthesis (Oster et al. 1998). The p33 open reading frame (ORF) is flanked by the viral 5' UTR and 3'CITE, and the sequence of both can be modified to examine their contribution to translation. The experimental p33-encoding

mRNA, a constitutively expressing p92 mRNA (which provides the viral RNAdependent RNA polymerase), and a reporter RNA replicon are simultaneously transfected into plant protoplasts. The more efficiently that p33 is translated from its message, the greater the level of replication and accumulation of the "reporter" TBSV RNA replicon (assayed by quantitative Northern blot analysis). Thus, the accumulation level of the RNA replicon provides a relative measure of the rate of translational of p33. This all-virus-based experimental mRNA system is favored over those using conventional reporter ORFs because the latter systems have been found to be refractory to the analysis of certain aspects of 3 'CITE activity (M.R. Fabian and K.A. White, unpubl.).

To assess the importance of the proposed Y-shaped structure of R3.5, different mutations were introduced into it that were designed to disrupt or maintain secondary structure, alter primary sequence, or remove bulges or internal loops. Sets of compensatory mutations in SL-B (ALT9, 10, 11 and ALT45, 46, 47), SL-C (ALT27, 28, 29, ALT30, 32, and ALT39, 40) and S-A (3/ 4S6-A, -B, -C and 3/4S5-A, -B, -C) all supported the importance of their proposed helical elements for translation (Fig. 3A). The deletion of bulges or internal loops in SL-C resulted in reduced translation ranging between $\sim 23 \%$ and $\sim 50 \%$ the level of wild type (ALT13, 14, 12, and 8, respectively), while similar deletions of the two internal loops in SL-B led to levels of $\sim 45 \%$ and $\sim 3 \%$ (ALT7 and 15 , respectively) (Fig. 3A). The two internal loops in S-A were also determined to be important since their deletion either abolished translation or reduced it to barely detectable levels (3/4B6 and 3/4B7, respectively) (Fig. 3A). Finally, the presence and identity of the residues in the predicted three-helix junction were also consequential since both types of modification significantly reduced translation levels to $\sim 3 \%-4 \%$ (ALT20 and 6) (Fig. 3A). Taken together, these results support the proposed Y-shaped secondary structure for R3.5 and showed that numerous bulges and internal loops are important features for optimal $3^{\prime} \mathrm{CITE}$ activity. 

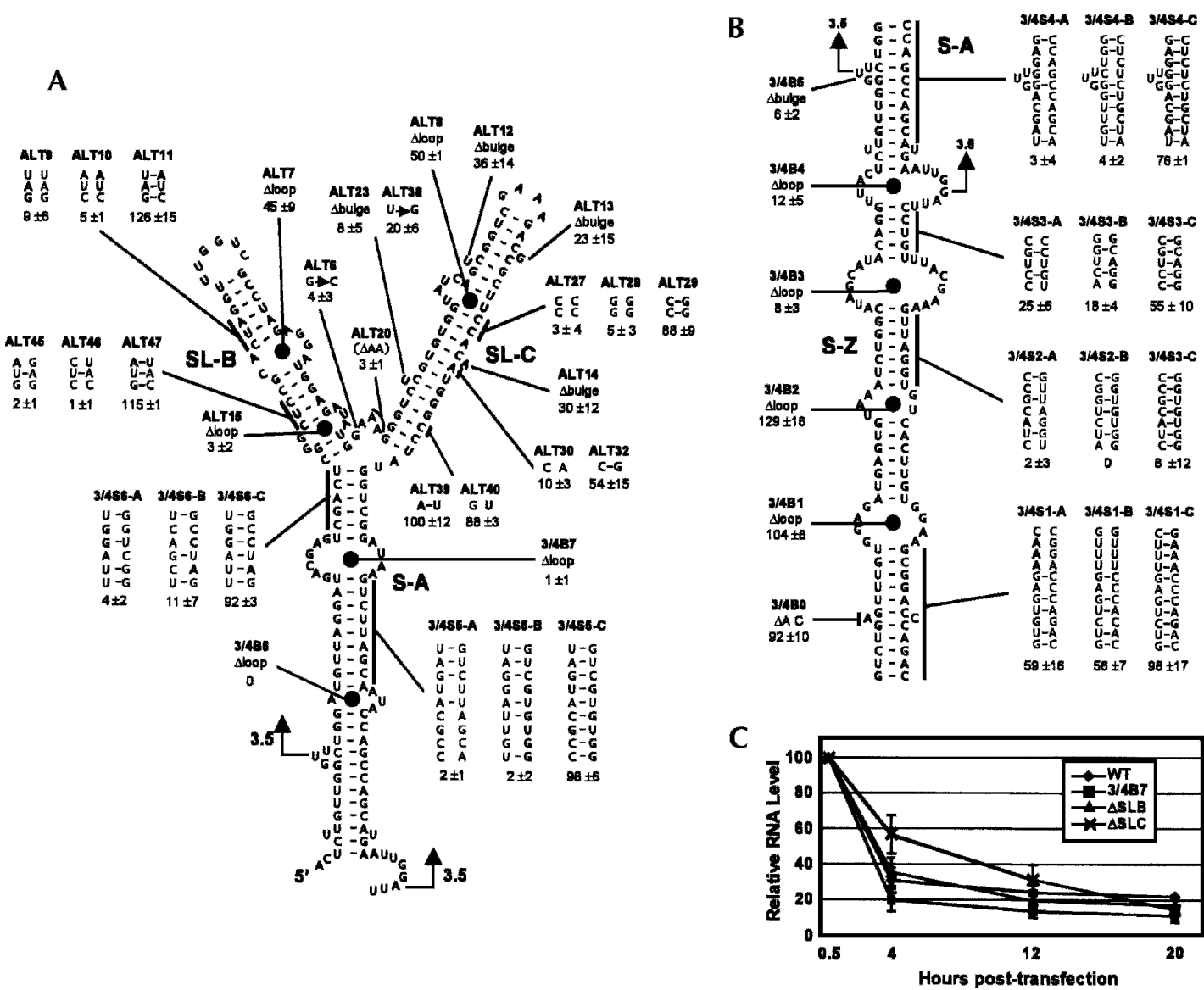

FIGURE 3. Defining the RNA secondary structure of R3.5 and surrounding regions important for translation. (A) Analysis of R3.5. Modifications to this region are shown and include relative translation levels determined using the viral-based translational assay system. Compensatory mutations of predicted base pairs or helical segments are indicated by a thick bar by the structure, whereas deletion of predicted internal loops are indicated by black circles within the structure. Substitutions or deletions are indicated by arrowheads showing the substitutions or the delta symbol $(\Delta)$ representing a deletion. The values represent relative DI RNA levels using the viral-based translational assay system. The means $( \pm \mathrm{SD})$ from three independent infections are presented, which were normalized to the levels of wild type (set as $100 \%)$. The $5^{\prime}$ and $3^{\prime}$ borders of R3.5 are indicated by arrows. (B) Analysis of structural features flanking R3.5. The $5^{\prime}$ and $3^{\prime}$ borders of R3.5 are indicated by arrows. Refer to $A$ for information on the different types of mutants and their analysis. (C) Stability assay of viral mRNAs. Normalized levels of viral mRNA remaining post-transfection are plotted over time. The values represent the averages from two independent experiments.

R3.5 represents the central portion of the $3^{\prime} \mathrm{CITE}$, and it is flanked by RIII and RIV (Fig. 1B). Previous deletion analysis of the $3^{\prime}$ CITE indicated that both RIII and RIV are important for its activity in vivo ( $\mathrm{Wu}$ and White 1999). Interestingly, mfold analysis of the $3^{\prime} \mathrm{CITE}$ suggested that the $3^{\prime}$ portion of RIII and the $5^{\prime}$ portion of RIV could interact with each other to form a single, long, primarily helical structure that extends below S-A, termed S-Z (Fig. $3 \mathrm{~B})$. The close proximity of this putative structure to the essential R3.5 core suggested that it could be important for 3'CITE activity. Indeed, disruption of the predicted helical regions of $\mathrm{S}-\mathrm{Z}$ reduced translational activity while, with one exception (3/4S2-A, -B, -C), restoration of base-pairing recovered translation to varying degrees (Fig. $3 \mathrm{~B}$ ). Deletion of some of the bulges and internal loops punctuating S-Z also negatively impacted translation (Fig. 4B). In general, for both double- and single-stranded regions, mutations in
S-Z that were located closer to R3.5 had more significant effects than did those positioned further away.

In vivo stability assays carried out with translationally defective experimental mRNAs that lacked SL-B or SL-C or that contained the B7 deletion in SL-A revealed rates of decay that were similar or lower than that of wild type (Fig. 4C). These results, combined with previous stability analyses (Wu and White 1999; Fabian and White 2004), suggest that the defects observed are primarily related to reduced levels of translation rather than accelerated degradation of mRNAs.

\section{Position-independent function of SL3 in the 5' UTR}

SL3 is the $5^{\prime}$-adapter in the $5^{\prime}-3^{\prime}$ interaction and is a subelement of the TSD (Fig. 1C). The TSD also plays a critical role in viral RNA replication as mutations in this 

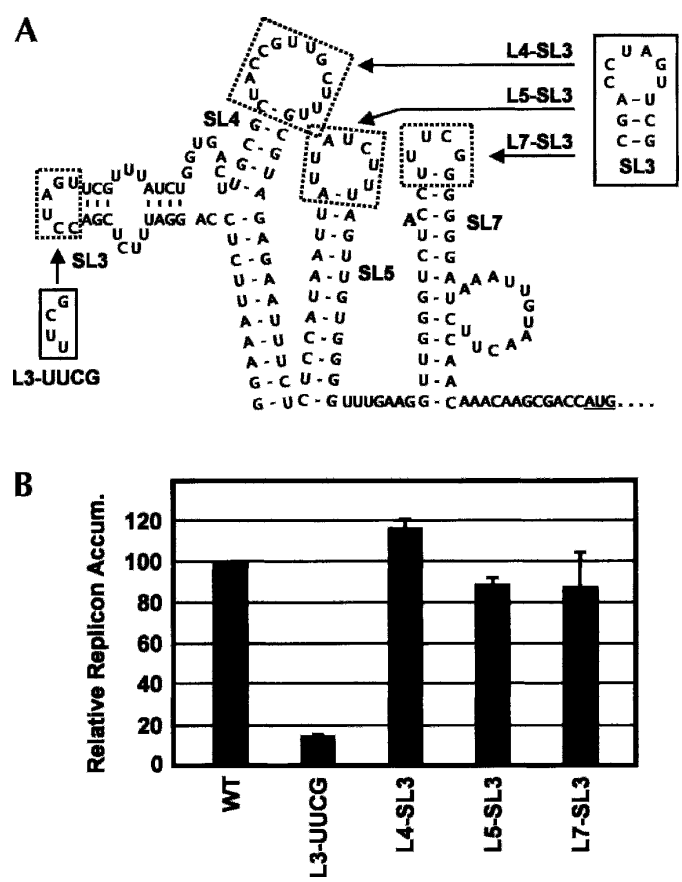

FIGURE 4. Positional analysis of $5^{\prime}$-adapter SL3 within the $5^{\prime}$ UTR. (A) RNA secondary structure of the TBSV 5' UTR showing mutants generated. Sequences boxed with dotted lines were substituted with the sequences boxed by solid lines. In addition to their SL3 substitutions (on the right), mutants L4-SL3, L5-SL3, and L7-SL3 also contained the L3-UUCG mutation (on the left). The underlined AUG represents the start codon for $\mathrm{p} 33$ translation in the viral mRNA. (B) Relative translation levels of mutants in $A$ as measured by the accumulation levels of the DI RNA replicon using the viral-based translational assay system. The values represent means $( \pm S D)$ from three independent infections.

domain severely compromise viral replicon accumulation in vivo (Wu et al. 2001; Ray et al. 2003, 2004). Translation and replication functions are thus highly integrated within this $5^{\prime}$-proximal RNA domain. Two other important RNA structures also reside in the TBSV $5^{\prime}$ UTR, and both are important for RNA replication. Just $3^{\prime}$ to the TSD is a simple hairpin, SL5, which separates the TSD from a second functional domain, the DSD (Fig. 1C). The TSD and DSD interact functionally via a pseudoknot, PK-TD1, that forms between L4 in the TSD and a $3^{\prime}$-proximal sequence in the DSD (Fig. 1C; Ray et al. 2003). The complex higher-order RNA structure of the 5' UTR led us to wonder if altered positioning of SL3 within this context would affect its ability to facilitate translation.

First, SL3 activity in translation was severely compromised by replacing its loop with a UUCG tetraloop (L3-UUCG) (Fig. 4B; Fabian and White 2004). Next, while maintaining the mutated SL3, other terminal loops in the TSD (L4), SL5, and the DSD (L7) were individually replaced with wild-type SL3 sequence (L4-SL3, L5-SL3, and L7-SL3, respectively) (Fig. 4B). In all three cases, translational activity was restored to near wild-type levels, indicating a large degree of flexibility with respect to the position of the $5^{\prime}$-adaptor SL in the $5^{\prime}$ UTR (Fig. 4B).

\section{Translation involves ribosome scanning from the $5^{\prime}$ terminus}

Having established the position independence of SL3, we next wanted to know how ribosomes accessed the start codon. This was assessed by introducing initiation codons (out of frame with the p33 reporter ORF) at selected sites within the 5' UTR (Fig. 5A). Any initiation of translation at an upstream AUG (uAUG) would decrease p33 translation and indicate that ribosomes traversed that upstream site. The insertion of uAUGs was chosen for evaluating scanning instead of the alternative approach of using $5^{\prime}$-terminal or internal stable SL structures (Kozak 1989) for several reasons. First, the 5' UTR is a complex higher-order RNA element and the introduction of additional large structural
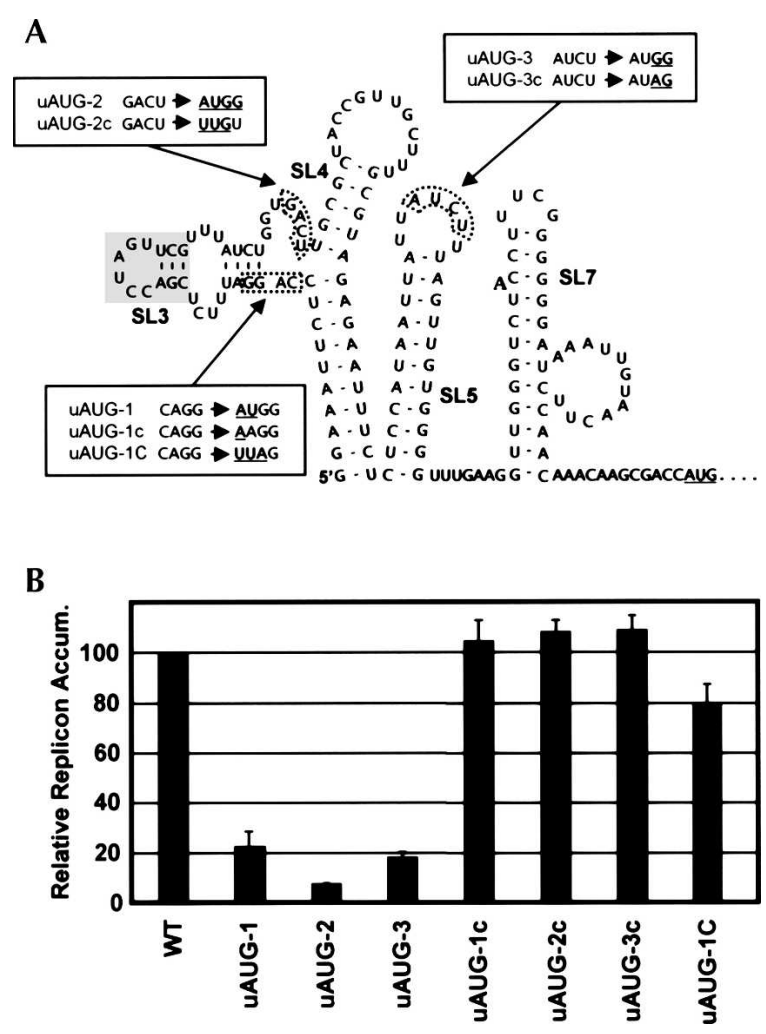

FIGURE 5. Effect of insertion of uAUGs in the 5' UTR. (A) RNA secondary structure of the TBSV $5^{\prime}$ UTR showing mutants generated. Sequences outlined with dotted lines were substituted with sequences that either did or did not introduce uAUGs (substituted nucleotides are underlined). The shaded stem-loop, SL3, represents the $5^{\prime}$-adapter in the $5^{\prime}-3^{\prime}$ RNA-RNA interaction. The underlined AUG represents the start codon for p33 translation in the viral mRNA. (B) Relative translation levels of mutants in $A$ as measured by the accumulation levels of the DI RNA replicon using the viral-based translational assay system. The values represent means $( \pm S D)$ from three independent infections. 
elements could interfere with its proper folding or sterically hinder the formation of the $5^{\prime}-3^{\prime}$ interaction. Second, since the translational process being studied is novel, we entertained the possibility that an internal initiation mechanism that was $5^{\prime}$-end-dependent could be operating. If this were the case, the results from the stable SL method would lead to erroneous conclusions. Third, the uAUG approach is more informative in that it can provide information on scanning at several different positions in the $5^{\prime}$ UTR, which can in turn offer insights into the path and processivity of ribosome migration.

Three different sites were selected for the introduction of uAUGs (Fig. 5A). The nucleotide substitutions made were carefully designed so as to allow for proper folding of the $5^{\prime}$ UTR, as determined by mfold analyses. In addition, corresponding control mutants were constructed in a similar manner, except that the substitutions made did not introduce uAUGs (Fig. 5A). The first uAUG was located $5^{\prime}$ to SL3 (uAUG-1), whereas the other two were positioned $3^{\prime}$ to SL3 (uAUG-2, -3). Analysis of these mutants revealed that all three uAUGs caused significant (approximately fivefold or greater) reductions in translational activity (Fig. 5B). In contrast, the control mutants (uAUG-1c, $-2 c,-3 c,-1 C$ ) showed only moderate differences compared to the wild type (Fig. 5B). The substantial inhibition that was observed only with the uAUGs suggests that a $5^{\prime}$-end scanning mechanism is used in the initiation process.

\section{DISCUSSION}

\section{Structure and function of the $3^{\prime}$ CITE}

Through mutational analysis we have defined a functionally relevant Y-shaped RNA secondary structure for the $3^{\prime} \mathrm{CITE}$. Our work verifies the existence of SL-B, the terminal loop of which is implicated in the $5^{\prime}-3^{\prime}$ interaction, and of SL-C, which was shown previously (via its deletion) to be essential for translational activity in vivo (Fabian and White 2004). The bottom of these two stems intersect with S-A to form a three-helix junction (Fig. 3A). Currently, the relative orientation of these helices is unknown and will require further structural analysis to uncover. However, the multiple functionally relevant internal loops and bulges present in all three of these helices (and also in S-Z) may contribute to tertiary interactions that dictate overall RNA topology. Alternatively, or additionally, these helical disruptions could be involved in binding to protein factors necessary for the translational process. Interestingly, $S-Z$, the newly defined extension of S-A, becomes less important at regions distal to R3.5. Although less significant, the lower portion of S-Z could facilitate and stabilize the formation of R3.5 and may act to extend R3.5 into solution for participation in RNA-RNA and RNA-protein interactions.

\section{Significance of the $5^{\prime}-3^{\prime}$ RNA-RNA interaction}

A central feature of the proposed translational mechanism for TBSV, and for related viruses such as BYDV, is the requirement for an $5^{\prime}-3^{\prime}$ RNA-RNA interaction in the message (Guo et al. 2001; Fabian and White 2004). In TBSV, this interaction is predicted to deliver translation factors bound by the $3^{\prime}$ CITE to the $5^{\prime}$ UTR (Fabian and White 2004). However, until now, this $5^{\prime}-3^{\prime}$ interaction has been supported only by sequence and genetic analyses (Fabian and White 2004). Here, we have provided formative physical evidence that these RNA elements do indeed interact with each other. Importantly, our data show that the complementary adapter SLs implicated in mediating this activity, SL3 and SL-B, are key determinants of complex formation. The role of these SLs is further supported by the fact that the interaction occurred with individually prefolded RNA transcripts. Thus, in their folded states, these SLs are predicted to be readily accessible, an attribute that is also likely to be important for their activity in vivo. An additional important point that can be deduced from this analysis is that the RNA complex can form without the requirement of protein factors. Although this suggests that the in vivo process may not require proteins to either initiate and/or stabilize the $5^{\prime}-3^{\prime}$ interaction, it does not preclude such involvement. However, the results do imply that the proposed binding of translational machinery to the $3^{\prime} \mathrm{CITE}$ is not an essential prerequisite for formation of the interaction.

In addition to the key adapter SL interaction, other contacts may also contribute to the binding energy, as suggested by low but detectable complex formation when heterologous SL pairs were tested (Fig. 2D). However, it is clear that SL-C is dispensable for binding since efficient complex formation occurred when it was deleted (Fig. 2B). Interestingly, this same SL-C deletion mutation was very defective when tested previously for translational activity in vivo (Fig. 2B; Fabian and White 2004). Taken together, these two findings suggest that the $5^{\prime}-3^{\prime}$ interaction alone is not sufficient to mediate efficient translation and that other factors related to SL-C are required. Currently, only the role of SL-B, at least the apical portion of it, is firmly established as the 3 '-adapter. However, the functions of the lower region of SL-B, as well as SL-C, S-A, and S-Z, remain unknown. As shown for the 3'TE of STNV (Gazo et al. 2004), and suggested for that of BYDV (Guo et al. 2001), these regions could be involved in binding protein factors that mediate recruitment of ribosomes. Indeed, a role in factor binding for SL-C would explain why translation is inhibited when SL-C is deleted, even though the $5^{\prime}-3^{\prime}$ interaction remains functional.

\section{Flexibility of $\mathbf{5}^{\prime}$-adaptor SL location}

For TBSV, we have shown that there is no strong position dependence for the $5^{\prime}$-adapter, SL3. That is, it can be 
re-located to different sites relative to either the $5^{\prime}$ terminus or the initiation codon in our experimental mRNA and still function efficiently in translation. However, within the viral genomic context, the normal location of SL3 is universally conserved among tombusviruses and is highly integrated with replication elements ( $\mathrm{Wu}$ et al. 2001; Ray et al. 2003, 2004). Thus, although flexible in terms of a translation-only context, the repositioning of SL3 in the wild-type genome would likely negatively affect the activity of other RNA elements involved in RNA replication and thus reduce the competitiveness of the virus.

The flexibility of the $5^{\prime}$-adapter position is also supported by its corresponding location in the $5^{\prime}$ UTR of TBSV sg mRNA1 (Fabian and White 2004). In sg mRNA1, an equivalent $5^{\prime}$-adapter is located at the very $5^{\prime}$ terminus of the message. This naturally occurring viral mRNA represents a 5'-extreme example in terms of adaptor position and further supports the concept of flexibility. The opposite extreme for $5^{\prime}$-adaptor location in a $5^{\prime}$ UTR would be to be positioned immediately adjacent to, or even $3^{\prime}$ to, the initiation codon. When we artificially introduced a uAUG $5^{\prime}$-proximal to SL3 in our experimental mRNA, this start codon effectively inhibited translation of $\mathrm{p} 33$, indicating that it was efficiently utilized by ribosomes (Fig. 5). This suggests that an adaptor located $3^{\prime}$ to an initiation codon can still function properly.

A natural example of extreme 3 '-proximal placement may in fact exist in Pelargonium line pattern virus (PLPV; genus unassigned, family Tombusviridae). Similar to all viruses in Tombusviridae, PLPV is uncapped and nonpolyadenylated and, as proposed for other members of this family, is thought to possesses a $3^{\prime} \mathrm{TE}$ (Fabian and White 2004; Castano and Hernandez 2005). This concept is supported by the observation that putative $5^{\prime}$ - and $3^{\prime}$-adaptors are present in both its genome and single sg mRNA (Fig. 6). In addition, the complementary sequences in these adaptors are all located in terminal loops of mfoldpredicted SLs, which would facilitate their proposed basepairing functions (Fig. 6). The PLPV genome is also unique in that it contains the shortest predicted 5' UTR (only $6 \mathrm{nt}$ long) of any sequenced member of Tombusviridae (Castano and Hernandez 2005). The proposed 5'-adaptor associated with the genome is of particular interest because the majority of the complementary sequence resides $3^{\prime}$ to the initiation codon (Fig. 6). If shown to be functionally relevant, this example would represent the most 3 '-proximal location for a naturally occurring $5^{\prime}$-adaptor module.

\section{Integration of $\mathbf{5}^{\prime}$-scanning into a translational model}

Our working model for the translation process in TBSV involves binding of translation initiation factors and/or the $43 \mathrm{~S}$ ribosomal subunit to RNA elements in the $3^{\prime}$ CITE and subsequent transfer of the $43 \mathrm{~S}$ subunit to the $5^{\prime}$ end of the

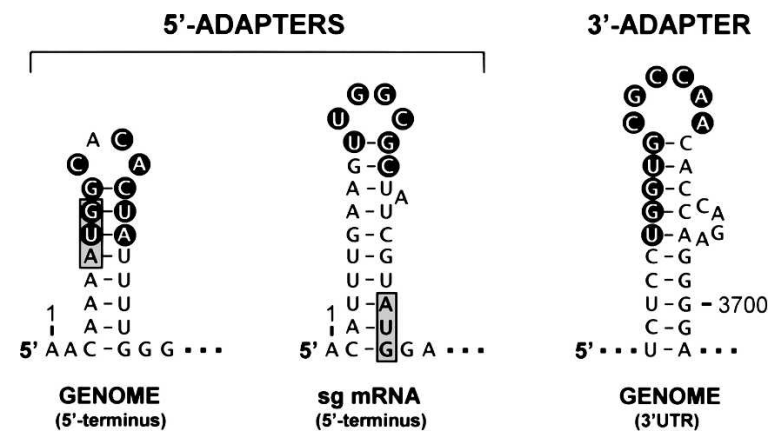

FIGURE 6. Proposed $5^{\prime}-3^{\prime}$ RNA-RNA interactions in Pelargonium line pattern virus. Mfold-predicted RNA secondary structures of $5^{\prime}$ - and $3^{\prime}$-adapter SLs in PLPV. Sequences predicted to interact via base-pairing are in white on solid black circles. Start codons in the $5^{\prime}$ UTR of the genome and subgenomic mRNA are boxed in gray.

message by the $5^{\prime}-3^{\prime}$ adaptor SL interaction (Fabian and White 2004). This model is similar to that proposed for BYDV (Guo et al. 2001) and may in fact apply to a large number of uncapped and nonpolyadenylated RNA viruses that possess potential $5^{\prime}-3^{\prime}$ RNA-RNA interactions and $3^{\prime}$ TEs (Fabian and White 2004).

Translation of most eukaryotic mRNAs involves $43 \mathrm{~S}$ subunit scanning from the $5^{\prime}$ terminus in a $3^{\prime}$ direction until it encounters an initiation codon, at which point ribosome assembly takes place and initiation begins (Kozak 2002). Recruitment of the $43 \mathrm{~S}$ subunit is facilitated by the $5^{\prime}$-cap structure and its associated initiation factors (Gebauer and Hentze 2004; Dreher and Miller 2006). For BYDV, studies using a stable SL structure introduced at a $5^{\prime}$-terminal or an internal region of the $5^{\prime}$ UTR suggested that $5^{\prime}$-scanning is necessary for ribosome loading (Guo et al. 2001). Similarly, our results with uAUGs suggest that the $43 \mathrm{~S}$ subunit scans from the $5^{\prime}$ end of the message (Fig. 5). In addition, the similar inhibitory effects of the out-of-frame uAUGs suggest that the scanning is relatively processive. However, because the TBSV $5^{\prime}$ UTR is very structured, this scanning process would likely require the assistance of a helicase. In plants, eIF4F (as opposed to eIFiso4F) is more effective at translating uncapped mRNAs, as well as messages with highly structured 5' UTRs (Gallie and Browning 2001). Therefore, if canonical initiation factors participate in this process, eIF4F represents a primary candidate for involvement.

The 5'-loading and scanning mechanism proposed also provides additional insights into other aspects of the translational process. TBSV transcribes two sg mRNAs during infections that are $3^{\prime}$-coterminal with the viral genome (Fig. 1). These smaller messages allow for efficient translation of $3^{\prime}$-proximally encoded ORFs that are translationally silent within the context of the viral genome. In TBSV, both sg mRNAs contain sequences in their 5' UTRs that are complementary to SL-B in the $3^{\prime} \mathrm{CITE}$ (i.e., sg mRNA 5'-adapters), thus similar functionally relevant 
$5^{\prime}-3^{\prime}$ interactions are predicted to be necessary for sg mRNA translation (Fabian and White 2004). Interestingly, even if the sg mRNA $5^{\prime}$-adapter sequences were able to interact with the $3^{\prime}$ CITE in the context of the genome, the proposed $5^{\prime}$-end loading mechanism for TBSV translation would not support initiation events at these internal sites. Preventing such events may be important, as spurious initiations within the viral genome could be deleterious to the virus by interfering with the timing and/or amount of 3 '-proximally encoded proteins produced-which, in TBSV, are controlled primarily at the level of sg mRNA transcription (Zhang et al. 1999; Qiu and Scholthof 2001; Choi and White 2002; Lin and White 2004).

Another fascinating aspect related to our results is that the scanning process is predicted to traverse the region that contains the $5^{\prime}$-adaptor SL. This concept is supported by the inhibitory effects of uAUGs positioned immediately upstream and downstream of SL3 (Fig. 5). One possibility is that scanning $43 \mathrm{~S}$ subunits are able to shunt around the interaction and thereby avoid disrupting it. Although plausible, this possibility does not seem likely based on our current understanding of the mechanism of shunting, which has specific sequence and structural requirements (Ryabova et al. 2002) that contrast the highly flexible context of the $5^{\prime}$-adaptor SL (Fig. 4). Instead, if scanning is indeed processive, then the $5^{\prime}-3^{\prime}$ interaction would be disrupted by $43 \mathrm{~S}$ subunit migration. In addition, if each translational initiation event requires loading of a subunit via the $5^{\prime}-3^{\prime}$ interaction, then the formation of this RNARNA bridge would have to be transitory-a property that could be facilitated by the relatively modest stability of the interaction. Considering this, a model for continuous $3^{\prime}$ CITE-mediated translation would therefore have to include the repeated formation and disruption of the $5^{\prime}-3^{\prime}$ interaction (Fig. 7). Such a process could also conceivably contribute to regulating the orderly loading of $43 \mathrm{~S}$ subunits at the $5^{\prime}$ end. Furthermore, this type of dynamic translational mechanism could apply to other viruses, such as BYDV, that utilize similar $5^{\prime}-3^{\prime}$ RNA-RNA interactions and are $5^{\prime}$-scanning dependent (Guo et al. 2001).

\section{MATERIALS AND METHODS}

\section{Viral constructs}

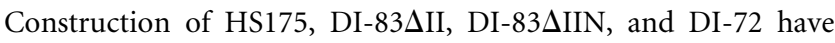
been described previously (Hearne et al. 1990; White and Morris 1994; Oster et al. 1998; Wu and White 1999; McCartney et al. 2005). All other mutant constructs used in this study were generated by modifying DI- $83 \Delta$ II. Mutants were generated by PCR-based oligonucleotide-mediated mutagenesis in combination with standard recombinant DNA cloning techniques. Each construct was sequenced across its entire PCR-derived segment to confirm that only the desired change was present.

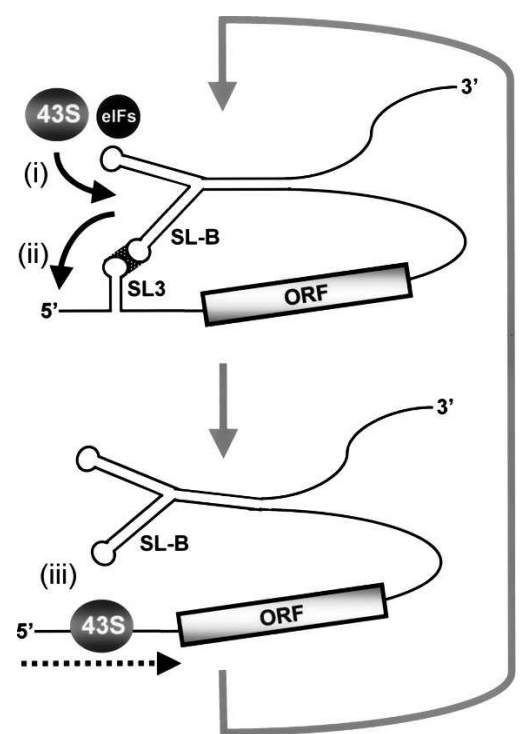

FIGURE 7. Model depicting the proposed dynamic nature of the 5 '-3' RNA-RNA interaction involved in TBSV translation. The TBSV 5' UTR and 3'CITE are shown schematically at either end of a coding region (ORF) represented by a box. For simplicity, SL3 is not shown in its more complex wild-type context of the TSD. The following steps are envisioned in the model. (i) The $43 \mathrm{~S}$ complex is recruited to the 3'CITE (possibly assisted by eIFs). (ii) The $43 \mathrm{~S}$ subunit is then delivered to the $5^{\prime}$ end of the mRNA via the base-pairing interaction between SL3 and SL-B. (iii) From the $5^{\prime}$ end, the $43 \mathrm{~S}$ complex proceeds to scan progressively in a $3^{\prime}$ direction and disrupts the basepairing interaction between SL3 and SL-B. Once the $43 \mathrm{~S}$ complex has cleared the 5' UTR, SL3 can refold and rebind to SL-B, thereby initiating a new translational cycle.

\section{Computer-aided analysis of viral RNA}

RNA secondary structures were predicted at $37^{\circ} \mathrm{C}$ by using Mfold version 3.1 (Mathews et al. 1999; Zuker et al. 1999).

\section{In vitro transcription}

Viral transcripts were synthesized in vitro by transcription of SmaI-linearized DNA using an Ampliscribe T7 RNA polymerase transcription kit (Epicentre Technologies) as described previously (Fabian and White 2004). Transcript concentrations were determined spectrophotometrically, and RNA integrity was verified by using agarose gel electrophoresis. RNA transcripts used for in vitro RNA-RNA gel shift assays were generated from gel-purified PCR fragments obtained using previously sequenced clones. 5' UTR and $3^{\prime}$ CITE transcripts were gel purified prior to use in binding experiments.

\section{RNA-RNA gel shift assay}

5' UTR transcripts were dephosphorylated by treatment with calf intestinal phosphatase and then end-labeled using $\gamma-{ }^{32} \mathrm{P}$-ATP and polynucleotide kinase. Labeled $5^{\prime}$ UTR transcripts $(30 \mathrm{nM})$ or unlabeled 3'CITE transcripts (ranging from 100-5000 nM) were incubated separately in a volume of $4 \mu \mathrm{L}$ RNA binding buffer (5 mM HEPES at $\mathrm{pH} 7.8,6 \mathrm{mM} \mathrm{MgCl}_{2}, 100 \mathrm{mM} \mathrm{KCl}, 3.8 \%$ glycerol, $0.1 \mu \mathrm{g}$ yeast RNA) for $5 \mathrm{~min}$ at $85^{\circ} \mathrm{C}$ and then slow 
cooled to $30^{\circ} \mathrm{C}$ to promote proper folding. The two transcripts were then combined and incubated for an additional $30 \mathrm{~min}$ at $30^{\circ} \mathrm{C}$. Following this incubation, tubes were cooled on ice for $10 \mathrm{~min}$, mixed with loading buffer (composed of an equal volume of RNA binding buffer and glycerol), and separated in a nondenaturing $10 \%$ polyacrylamide gel (supplemented with $6 \mathrm{mM}$ $\mathrm{MgCl}_{2}$ ) for $4.5 \mathrm{~h}$. Electrophoresis was carried out in standard $1 \times$ tris-borate buffer supplemented with $6 \mathrm{mM} \mathrm{MgCl}_{2}$ and was performed at $4^{\circ} \mathrm{C}$. Gels were subsequently dried and analyzed by radio-analytical scanning using an Instant Imager (Packard Instrument Co.). The apparent $\mathrm{K}_{\mathrm{d}}$ for the wild-type 5' UTR and wild-type $3^{\prime}$ CITE interaction was calculated from the binding curve generated by using nonlinear regression analysis. Complete binding was not achievable (probably because a fraction of the RNA was inactive due to misfolding and/or limitations related to the electrophoretic assay used); therefore, the $\mathrm{B}_{\max }$ (maximum bound) was calculated via best-fit extrapolation of the curve and the concentration of $5^{\prime}$ UTR at one-half $B_{\max }$ was defined as the apparent $\mathrm{K}_{\mathrm{d}}$.

\section{In vivo translation assays}

Translation assays were carried out as described previously (Fabian and White 2004). Briefly, protoplasts were prepared from 6- to 8-d-old cucumber cotyledons and transfected, using polyethylene glycol- $\mathrm{CaCl}_{2}$, with viral RNA transcripts. Each transfection contained $1 \mu \mathrm{g}$ for DI-72 reporter replicon, $5 \mu \mathrm{g}$ of HS175 (mRNA encoding p92), and $5 \mu \mathrm{g}$ DI-83 II (experimental mRNA encoding p33). Transfections were incubated in a growth chamber under fluorescent lighting for $22-24 \mathrm{~h}$ at $22^{\circ} \mathrm{C}$. The relative level of p33 translation was monitored by assessing the accumulation level of DI-72 by Northern blot analysis.

Total nucleic acids were harvested from transfected protoplasts as described previously (White and Morris 1994). One-fifth of the total nucleic acid was separated in denaturing $4.5 \%$ polyacrylamide gels containing $8 \mathrm{M}$ Urea. Gels were stained with ethidium bromide to ensure even loading of samples. Northern blot analysis of viral plus-strand accumulation was conducted by transferring total nucleic acid to nylon, followed by hybridization with a ${ }^{32} \mathrm{P}$-end-labeled oligonucleotide probe complementary to the 3 -terminal $23 \mathrm{nt}$ of the TBSV genome. The intensity of the hybridization signal was quantified by radio-analytical scanning using an Instant Imager (Packard Instrument Co.) and is represented numerically or graphically with standard deviations (SDs) derived from three independent experiments.

\section{RNA stability assays}

Analysis of viral mRNA in vivo stability was performed as described previously (Fabian and White 2004). Briefly, protoplasts

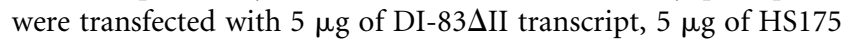
transcript, and $1 \mu \mathrm{g}$ of DI-72 transcript and subsequently treated with RNase A (final concentration $10 \mu \mathrm{g} / \mathrm{mL}$ ) to removed transcripts outside of the protoplasts. Total nucleic acids were extracted from protoplasts at $0.5,4,12$, and $20 \mathrm{~h}$ post-inoculation, and mRNA levels were analyzed by Northern blotting.

\section{ACKNOWLEDGMENTS}

We thank members of our laboratory for reviewing the manuscript and Geetha Mahalingam and Logan Donaldson for technical assistance. M.R.F. was supported by an NSERC postgraduate scholarship. This research was supported by NSERC, PREA, and CRC.

Received February 20, 2006; accepted March 30, 2006.

\section{REFERENCES}

Castano, A. and Hernandez, C. 2005. Complete nucleotide sequence and genome organization of Pelargonium line pattern virus and its relationship with the family Tombusviridae. Arch. Virol. 150: 949965.

Choi, I.R. and White, K.A. 2002. An RNA activator of subgenomic mRNA1 transcription in tomato bushy stunt virus. J. Biol. Chem. 277: 3760-3766.

Danthinne, X., Seurinck, J., Meulewaeter, F., Van Montagu, M., and Cornelissen, M. 1993. The $3^{\prime}$ untranslated region of satellite tobacco necrosis virus RNA stimulates translation in vitro. Mol. Cell. Biol. 13: 3340-3349.

Dreher, T.W. and Miller, W.A. 2006. Translational control in positive strand RNA plant viruses. Virology 344: 185-197.

Fabian, M.R. and White, K.A. 2004. 5'-3' RNA-RNA interaction facilitates cap- and poly(A) tail-independent translation of tomato bushy stunt virus mRNA: A potential common mechanism for Tombusviridae. J. Biol. Chem. 279: 28862-28872.

Gallie, D.R. and Browning, K.S. 2001. eIF4G functionally differs from eIFiso4G in promoting internal initiation, cap-independent translation, and translation of structured mRNAs. J. Biol. Chem. 276: 36951-36960.

Gazo, B.M., Murphy, P.A., Gatchel, J.R., and Browning, K.S. 2004. A novel interaction of cap-binding protein complexes eIF4F and eIF(iso) $4 \mathrm{~F}$ with a region in the $3^{\prime}$ untranslated region of satellite tobacco necrosis virus. J. Biol. Chem. 279: 13584-13592.

Gebauer, F. and Hentze, M.W. 2004. Molecular mechanisms of translational control. Nat. Rev. Mol. Cell Biol. 5: 827-835.

Guo, L., Allen, E., and Miller, W.A. 2000. Structure and function of a cap-independent translation element that functions in either the $3^{\prime}$ or the $5^{\prime}$ untranslated region. RNA 6: 1808-1820.

- 2001. Base-pairing between untranslated regions facilitates translation of uncapped, nonpolyadenylated viral RNA. Mol. Cell 7: 1103-1109.

Hearne, P.Q., Knorr, D.A., Hillman, B.I., and Morris, T.J. 1990. The complete genome structure and synthesis of infectious RNA from clones of tomato bushy stunt virus. Virology 177: 141-151.

Kneller, E.L., Rakotondrafara, A.M., and Miller, W.A. 2005. Capindependent translation of plant viral RNAs. Virus Res. (in press).

Koh, D.C., Liu, D.X., and Wong, S.M. 2002. A six-nucleotide segment within the 3' untranslated region of hibiscus chlorotic ringspot virus plays an essential role in translational enhancement. J. Virol. 76: 1144-1153.

Kozak, M. 1989. Circumstances and mechanisms of inhibition of translation by secondary structure in eucaryotic mRNAs. Mol. Cell. Biol. 9: 5134-5142.

- 2002. Pushing the limits of the scanning mechanism for initiation of translation. Gene 299: 1-34.

Lin, H. and White, K.A. 2004. A complex network of RNA-RNA interactions controls subgenomic mRNA transcription in a Tombusvirus. EMBO J. 23: 3365-3374.

Mathews, D.H., Sabina, J., Zuker, M., and Turner, D.H. 1999. Expanded sequence dependence of thermodynamic parameters improves prediction of RNA secondary structure. J. Mol. Biol. 288: 911-940.

McCartney, A.W., Greenwood, J.S., Fabian, M.R., White, K.A., and Mullen, R.T. 2005. Localization of the tomato bushy stunt virus replication protein $\mathrm{p} 33$ reveals a peroxisome-to-endoplasmic reticulum sorting pathway. Plant Cell 17: 3513-3531.

Mizumoto, H., Tatsuta, M., Kaido, M., Mise, K., and Okuno, T. 2003. Cap-independent translational enhancement by the 
$3^{\prime}$ untranslated region of red clover necrotic mosaic virus RNA1. J. Virol. 77: 12113-12121.

Oster, S.K., Wu, B., and White, K.A. 1998. Uncoupled expression of p33 and p92 permits amplification of tomato bushy stunt virus RNAs. J. Virol. 72: 5845-5851.

Pelletier, J. and Sonenberg, N. 1988. Internal initiation of translation of eukaryotic mRNA directed by a sequence derived from poliovirus RNA. Nature 334: 320-325.

Qiu, W. and Scholthof, H.B. 2001. Effects of inactivation of the coat protein and movement genes of Tomato bushy stunt virus on early accumulation of genomic and subgenomic RNAs. J. Gen. Virol. 82: 3107-3114.

Qu, F. and Morris, T.J. 2000. Cap-independent translational enhancement of turnip crinkle virus genomic and subgenomic RNAs. J. Virol. 74: 1085-1093.

Ray, D., Wu, B., and White, K.A. 2003. A second functional RNA domain in the $5^{\prime}$ UTR of the tomato bushy stunt virus genome: Intra- and interdomain interactions mediate viral RNA replication. RNA 9: 1232-1245.

Ray, D., Na, H., and White, K.A. 2004. Structural properties of the $5^{\prime}$ terminal T-shaped domain that promote efficient TBSV RNA Replication. J. Virol. 78: 10490-10500.

Ryabova, L.A., Pooggin, M.M., and Hohn, T. 2002. Viral strategies of translation initiation: ribosomal shunt and reinitiation. Prog. Nucleic Acid Res. Mol. Biol. 72: 1-39.

Scholthof, K.B., Scholthof, H.B., and Jackson, A.O. 1995. The tomato bushy stunt virus replicase proteins are coordinately expressed and membrane associated. Virology 208: 365-369.

Shen, R. and Miller, W.A. 2004. The $3^{\prime}$ untranslated region of tobacco necrosis virus RNA contains a barley yellow dwarf virus-like capindependent translational element. J. Virol. 78: 4655-4664.

Timmer, R.T., Benkowski, L.A., Schodin, D., Lax, S.R., Metz, A.M., Ravel, J.M., and Browning, K.S. 1993. The 5' and $3^{\prime}$ untranslated regions of satellite tobacco necrosis virus RNA affect translational efficiency and dependence on a 5' cap structure. J. Biol. Chem. 268: 9504-9510.

Wang, S. and Miller, W.A. 1995. A sequence located 4.5 to 5 kilobases from the $5^{\prime}$ end of the barley yellow dwarf virus (PAV) genome strongly stimulates translation of uncapped mRNA. J. Biol. Chem. 270: $13446-13452$.

Wang, S., Browning, K.S., and Miller, W.A. 1997. A viral sequence in the $3^{\prime}$-untranslated region mimics a $5^{\prime}$ cap in facilitating translation of uncapped mRNA. EMBO J. 16: 4107-4116.

Wells, S.E., Hillner, P.E., Vale, R.D., and Sachs, A.B. 1998. Circularization of mRNA by eukaryotic translation initiation factors. Mol. Cell 2: 135-140.

White, K.A. and Morris, T.J. 1994. Nonhomologous RNA recombination in tombusviruses: Generation and evolution of defective interfering RNAs by stepwise deletions. J. Virol. 68: 14-24.

White, K.A. and Nagy, P.D. 2004. Advances in the molecular biology of Tombusviruses: Gene expression, genome replication and recombination. Prog. Nucleic Acid Res. Mol. Biol. 78: 188-226.

$\mathrm{Wu}$, B. and White, K.A. 1999. A primary determinant of capindependent translation is located in the $3^{\prime}$-proximal region of the tomato bushy stunt virus genome. J. Virol. 73: 8982-8988.

Wu, B., Vanti, W.B., and White, K.A. 2001. An RNA domain within the $5^{\prime}$ untranslated region of the tomato bushy stunt virus genome modulates viral RNA replication. J. Mol. Biol. 305: 741-756.

Zhang, G., Slowinski, V., and White, K.A. 1999. Subgenomic mRNA regulation by a distal RNA element in a (+)-strand RNA virus. RNA 5: 550-561.

Zuker, M., Mathews, D.H., and Turner, D.H. 1999. Algorithms and thermodynamics for RNA secondary structure prediction: A practical guide. In RNA biochemistry and biotechnology (eds. J. Barciszewski and B.F.C. Clark), pp. 11-43. NATO ASI Series, Kluwer Academic Publishers, Boston. 

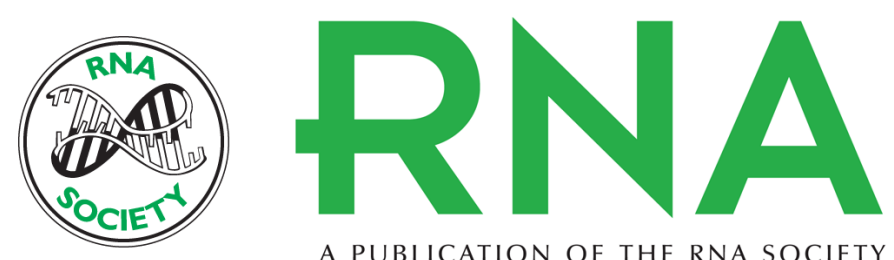

A PUBLICATION OF THE RNA SOCIETY

\section{Analysis of a 3'-translation enhancer in a tombusvirus: A dynamic model for RNA-RNA interactions of mRNA termini}

Marc R. Fabian and K. Andrew White

RNA 2006 12: 1304-1314

References

License

Email Alerting Service
This article cites 36 articles, 21 of which can be accessed free at:

http://rnajournal.cshlp.org/content/12/7/1304.full.html\#ref-list-1

Receive free email alerts when new articles cite this article - sign up in the box at the top right corner of the article or click here.

To subscribe to RNA go to:

http://rnajournal.cshlp.org/subscriptions 